\section{Optimization of primary hepatocyte isolation for the pharmacological characteriza- tion of metabotropic glutamate receptor (mGluR) s ubtype 5: A study on Reduction}

\author{
Clarissa Berardo, Andrea Ferrigno, \\ Veronica Siciliano, Marta Cagna, \\ Plinio Richelmi, Mariapia Vairetti, \\ Laura G. Di Pasqua \\ Department of Internal Medicine and \\ Therapeutics, University of Pavia, Pavia, \\ Italy
}

\begin{abstract}
To minimize the number of animals used during experiments, it is important to choose the suitable enzyme according to the final goal. In our work, we demonstrated the superiority of collagenase IV in the maintenance of functional transmembrane receptor, thus the pharmacological activity, in isolated rat hepatocytes.
\end{abstract}

\section{Introduction}

In our previous studies in rat hepatocytes, 1,2 a significant variability in the responsiveness of mGlu 5 receptor has been found among different hepatocyte preparations, forcing us to increase the number of rats to obtain statistically significant results. The most used enzyme in hepatocyte isolation is collagenase Type I from Clostridium hystoliticum. It should be noted that the majority of pharmacological studies on isolated hepatocytes are aimed to evaluate the function of intracellular cytochromes; on the contrary, mGluR5 is a transmembrane receptor. Our hypothesis was that collagenase I partially affected the function of mGluR5. The goal of this research was to compare mGluR5 expression and function in hepatocytes obtained from collagenases type I and type IV.

\section{Materials and Methods}

Hepatocytes from male Wistar rats (200-250 g) were isolated using collagenases I or IV (Worthington). After isolation, morphology, cell number and viability of rat hepatocytes were measured. mGluR5 protein expression was assessed by western blot analyses. mGluR5 activation was evaluated by inositol monophosphate (IP-1, Cisbio Kit) accumulation after treatment with the mGluR5 orthosteric agonist ACPD (Tocris) and the selective antagonist MPEP (Tocris).

\section{Results}

No difference in morphology, cell number and viability was observed when using collagenase I as compared with collagenase IV. A significant increase in mGluR5 protein expression was observed in hepatocytes isolated using collagenase IV with respect to collagenase I. Moreover, hepatocytes treated with ACPD and isolated using collagenase I presented lower levels of IP-1 when compared to the hepatocytes isolated by collagenase IV; in addition, hepatocytes isolated by collagenase IV and MPEPtreated showed a significant decrease in IP1 with respect to hepatocytes isolated with collagenase I.

\section{Discussion and Conclusions}

According to our findings, rat hepatocytes isolated with collagenase IV are more suitable for pharmacological studies on mGlu5 receptor. These results suggest that collagenase IV better preserve the functionality of surface proteins. Collagenase I has been widely used for hepatocyte preparations for studies on hepatic cytochromes; cytochromes, being intracellular enzymes, are probably not affected by the collagenase used during the isolation process.

The use of collagenase IV may result in a higher consistency in the results, reducing the number of animals, especially in pharmacological studieson surface receptors.
Correspondence: Clarissa Berardo, Department of Internal Medicine and Therapeutics, University of Pavia, Pavia, Italy. E-mail: clarissa.berardo01@universitadipavia.it

Key words: Reduction; mGluR5; collagenase; rat hepatocytes; MPEP.

Acknowledgments: This work was carried out using the internal resources of the Department of Internal Medicine and Therapeutics, Cellular and Molecular Pharmacology and Toxicology Unit.

Disclosures: All the authors have nothing to disclose.

Conference presentation: This paper was presented at the Third Centro 3R Annual Meeting - L'era delle 3R: modelli in silico, in vitro e in vivo per promuovere la ricerca traslazionale 30 September - 1 October 2021, Evento online organizzato dal Politecnico di Torino.

Received for publication: 9 July 2021.

Accepted for publication: 7 September 2021.

This work is licensed under a Creative Commons Attribution NonCommercial 4.0 License (CC BY-NC 4.0).

\section{(C) Copyright: the Author(s), 2021}

Licensee PAGEPress, Italy

Biomedical Science and Engineering 2021; 4(s1):151 doi:10.4081/bse.2021.151

\section{References}

1. Storto M, de Grazia U, Knöpfel T, et al. Selective blockade of mGlu5 metabotropic glutamate receptors protects rat hepatocytes against hypoxic damage. Hepatology 2000;31:649-55.

2. Ferrigno A, Berardo C, Di Pasqua LG, et al. Selective Blockade of the Metabotropic Glutamate Receptor mGluR5 Protects Mouse Livers in In Vitro and Ex Vivo Models of Ischemia Reperfusion Injury. Int $\mathrm{J}$ Mol Sci 2018;19:314. 